\title{
Irritation und Phantasie
}

\section{ZfE}

\section{Zur Möglichkeit von Erfahrungen in schulischen Lernprozessen}

\author{
Arno Combe • Ulrich Gebhard
}

Zusammenfassung: In Erfahrungsprozessen sind wir auf intensive Weise in eine Situation oder auch - für schulisches Lernen besonders wichtig - in die Auseinandersetzung mit einem Lerngegenstand verwickelt. Wir werden in diesem Artikel darstellen, wie solche sinnhaften, persönlichkeitswirksamen und allenthalben geforderten Lernprozesse vor dem Hintergrund einer Theorie des Erfahrungslernens gedacht werden können. Erfahrungen konstituieren sich innerhalb eines Prozesses der Krisenbewältigung. Dies darf nicht als eine negativistische Sicht missverstanden werden. Wir werden zeigen, wie Krisen und Irritationen in konstruktive Lernprozesse transformiert werden und als „fruchtbare Momente in Bildungsprozessen“ fungieren können. Allerdings sind zentrale prozessuale und strukturelle Momente des Erfahrungsprozesses wie Widerstand, Intuition, Imagination und Phantasie in Bezug auf den schulischen Lernprozess nicht eigentlich erschlossen worden. An dieser Leerstelle setzt der Aufsatz an, denn vor allem im Falle der von einer Irritation inspirierten Phantasie wird deren Potenzial ersichtlich, Mittelpunkt und dynamisches Medium eines Wechselverkehrs zwischen innerer und äußerer Welt und entsprechender Übergänge zwischen Ich und Gegenstand zu sein. Diese These wird im Hinblick auf ihre empirisch-psychologische und didaktische Anschlussfähigkeit thematisiert. Schließlich wird mit dem Konzept der „Alltagsphantasien“ gezeigt, dass und wie Irritation und Phantasie in Bildungsprozessen fruchtbar mit reflexiven Vorgängen verbunden werden können.

Schlüsselwörter: Erfahrung · Phantasie · Intuition · Irritation · Fruchtbarer Moment im Bildungsprozess

\section{Irritation and fantasy - The possibility of learning by experience in academic settings}

\begin{abstract}
In processes of experience, we become intensely involved in a situation or-what is particularly important for academic learning-in the examination of a learning object. In this article we show in which ways meaningful, ubiquitous learning processes that embrace the individual can be understood against the background of a theory of learning based on experience. Experience constitutes itself within a process of crisis management which must not be misunderstood as a negativistic perspective. For we will demonstrate how crises and irritation can be transformed
\end{abstract}

Online publiziert: 23.12 .2009

(C) Die Autoren 2009. Dieser Artikel ist auf Springerlink.com mit Open Access verfügbar.

Univ.-Prof. em. Dr. A. Combe $(\bowtie) \cdot$ Univ.-Prof. Dr. U. Gebhard

Fakultät für Erziehungswissenschaft, Psychologie und Bewegungswissenschaft, Universität

Hamburg, Von-Melle-Park 8, 20947 Hamburg, Deutschland

E-Mail: Arno.Combe@gmx.de; Ulrich.Gebhard@uni-hamburg.de 
into constructive learning processes and how they function as "fruitful elements in educational processes." However, key processual and structural elements of the process of experience, such as resistance, intuition, imagination, and fantasy, have in effect not yet been developed in learning theory, at least with respect to the academic learning process. It is precisely this gap that this essay acts on. Particularly in the case of fantasy inspired by irritation, its potential of being the focus and dynamic medium of intercommunication between the inner and outer world, and of the corresponding transitions between self and object, becomes evident. This assumption will be thematized with respect to its empirical-psychological and didactic relevance. Finally, using the concept of everyday fantasies it will be demonstrated that and how irritation and fantasy can be fruitfully combined with reflective processes.

Keywords: Experience $\cdot$ Fantasy $\cdot$ Fruitful element in the educational process - Intuition · Irritation

\section{Sinn und Erfahrung in schulischen Lernprozessen}

Wirklich intensive, die innere Beteiligung der Person erreichende Lernprozesse haben in der Schule Seltenheitswert. Es gibt diese nur als „Inseln der Intensität in einem Meer der Routine“ (Ziehe 1996, S. 940). Das schulische Lernen sei allzu sehr vom Pflichtcharakter durchzogen, so Ulrich Oevermann. Das Potenzial an Neugier und Phantasie, das Kinder mitbringen, werde verschenkt. Oevermann (1996) fordert die Abschaffung der Schulpflicht (kritisch hierzu: Combe 2005). Wenn man für eine Abkehr vom Verschulten plädiert, so muss doch die Hoffnung geschwunden sein, in der Schule ließe sich noch in einem biografisch bedeutsamen Sinne lernen. Dennoch warnt etwa Jürgen Baumert (2008) davor, die Schule schlecht zu reden. Als gesellschaftliche Organisationsform einer Bildung für alle habe sie nicht versagt. Gleichwohl leide sie unter einem chronischen Motivationsproblem. Die zwar schwierige, aber nicht unlösbare Aufgabe der Lehrer und Lehrerinnen sei es, die Schüler wenigstens situativ zum Lernen zu „,verführen“ (Baumert 2008, S. 21).

Die Frage, ob das Lernen in der Schule biografische Tiefenschichten bei Schülern zu erreichen vermag, soll hier vor allem grundsätzlich und nur im Ansatz in Hinblick auf seine mögliche schulpraktische Verwirklichung diskutiert werden. Was ist eigentlich gemeint, wenn von biografisch bedeutsamem und sinnhaftem Lernen die Rede ist? Wir müssten zunächst mehr über die Verknüpfungsmöglichkeiten von Sinn und Lernen wissen, über Prozesse, die den Verständnishorizont, mit dem wir der Welt und uns selbst begegnen, nicht nur erweitern, sondern verwandeln.

Es gibt eine Dimension, die auf derartig persönlichkeitswirksame Lernprozesse verweist: der Prozess der Erfahrung (vgl. zu verschiedenen Zugängen: Buck 1967; Kambartel 1972; Meyer-Drawe 1996, 2005; Waldenfels 2002; Benner 2005; Combe 2004, 2006; Combe \& Gebhard 2007). Erfahrungen sind es, aus denen das Subjekt immer wieder als ein anderes hervorgeht. In Erfahrungsprozessen sind wir auf vielschichtige und höchst intensive Weise in eine Auseinandersetzung mit einer Situation oder auch - für Lernprozesse besonders wichtig - mit einem Sachverhalt verwickelt. Mit unserem Verständnis von Erfahrungsprozessen verbunden ist der Vorgang einer sich fortentwickelnden Wechselwirkung zwischen Subjekt und dem Objekt der Erfahrung. In besonders hellsichtigen 
Momenten dieses Prozesses registrieren wir einen Wandel in unserer Beziehung zum Gegenstand und damit auch zu uns selbst. Wir werden in diesem Aufsatz entwickeln, wie intensive, persönlichkeitswirksame Lernprozesse vor dem Hintergrund einer Erfahrungstheorie des Lernens gedacht werden können.

Selbstverständlich kann man skeptisch sein, ob der Anspruch, Lernen auf Erfahrung hin anzulegen, in der Schule überhaupt eingelöst werden kann. Trotz dieser nicht unberechtigten Skepsis gehen wir von einem grundlegenden Sinnbedürfnis der Subjekte aus, das auch für den Bereich des schulischen Lernens gelten muss und das nur um den Preis sinnentleerten Lernens aufgegeben werden kann. Wenn es den Subjekten immer wieder gelingt, Lernprozesse mit einer Erfahrungsbewegung zu verbinden, wenn es schließlich den Subjekten zusätzlich gelingt, ihre Auseinandersetzung mit (Lern-)Gegenständen als sinnvoll zu interpretieren, können persönlichkeitswirksame Lernprozesse stattfinden.

In der Geschichte des Erfahrungskonzeptes lassen sich unterschiedliche Linien herausarbeiten. Wir beschränken uns hier nicht darauf, Erfahrungen, wie etwa in der hermeneutischen Traditionslinie, z. B. bei Gadamer (1960), vor allem auf sprachlich-kognitiver Ebene anzusiedeln. Vielmehr betonen wir die Vielschichtigkeit, mit der wir bei einem Erfahrungsprozess in einen Sachverhalt verwickelt sein können: Diese reicht vom leibnahen, affektiv-unmittelbaren Einbezogensein über die Dynamik von Phantasieprozessen bis zu sprachlichen Gestaltgebungsversuchen. Erfahrungen fordern damit letztlich auch zur Selbstreflexion heraus. Erfahrungen-Machen bedeutet also, dass der Einzelne auf der Ebene der leiblich-sinnlichen Positionalität seiner Praxis, sozusagen als körperlich engagiertes Individuum involviert ist (vgl. Meyer-Drawe 2005, S. 512). Diese Dimension berührt neben der sprachlich-reflexiven ausdrücklich auch die Ebene der Anmutung und Intuition - ein Umstand, den wir im Ansatz der Alltagsphantasien (Gebhard 2007) aufnehmen werden (s. Abschnitt 4).

Wir rücken bei der folgenden Darstellung drei Aspekte in den Vordergrund, die wir in ihrer Verflechtung als konstitutiv für Erfahrungsprozesse betrachten:

1. die krisenhafte, irritierende Ausgangskonstellation,

2. der Umgang mit damit verbundenen Lernwiderständen und

3. die Rolle und das Gewicht der Phantasie.

Wir beziehen uns dabei zunächst auf die Annahme der pragmatistischen Erfahrungstheorie, nach der persönlichkeitswirksame Erfahrungen oft in einer Konstellation der Krise und ausgehend vom Zustand der Irritation gemacht werden. Eine Reihe von philosophischen und bildungstheoretischen Positionen verweisen auf diese irritierende, krisenhafte, ja negative Seite von Erfahrungsprozessen (vgl. Gadamer 1960, S. 383; Buck 1967; Benner 2005; Dewey 1988, 2002). Schon Dewey (1988, S. $80 \mathrm{ff}$.) beschreibt den Beginn des Erfahrungsprozesses als Irritation (vgl. auch English 2005). Der Anfang des Erfahrungsprozesses enthält dabei einen Moment des „Widerfahrnisses“ (vgl. Bollnow 1968; Waldenfels 2002). Im Begriff der Negativität, der schließlich bei Buck (1967) in den Vordergrund rückt, steckt vor allem die Annahme, dass es zu Beginn des Erfahrungsprozesses in gewissem Sinne zu einer Negation, mindestens jedoch zu einer Verfremdung des bisherigen Wissens und Könnens kommt. In der Philosophie werden mit dem Begriff der Negativität oft Prozesse angezeigt, die mit einer schmerzhaften Umkehr und mit einem „Umlernen“ verbunden sind (Buck 1967). 
Durch diese Anbindung des Erfahrungsprozesses an krisenhafte Ausgangskonstellationen entstehen für das schulische Lernen Fragen: Wie viel Befremdung, wie viel Konfrontation und Irritation, Konflikt und Diskontinuität verträgt der Unterricht? Und: Liegt hier nicht eine allzu negative Dramatisierung von Bildungsprozessen vor?

Vor diesem Hintergrund muss geklärt werden - das halten wir für einen entscheidenden Punkt, da ansonsten die Begriffe „Krise“ und „Irritation“ negativ konnotiert sein würden - ob und wie Irritationen auch fruchtbar werden können. Irritationen könnten zumindest auch in den immer wieder zitierten, jedoch wenig empirisch erforschten ,fruchtbaren Moment im Bildungsprozess“ (Copei 1969) führen. Copei sagt, dass es einer „Triebfeder", einer Spannung, eben einer krisenhaften Konstellation bedürfe, um mehr als nur eine ,gedächtnismäßige Einprägung überlieferter Sinngehalte“ (Copei 1969, S. 102) zu bewirken. In solchen Momenten leuchte - so Copei - die Erkenntnis zwar auf, sei aber als solche noch unfertig. Genau diesen Zustand und seine Transformationen wollen wir in den Blick nehmen. Für einen sich fortentwickelnden Prozess der Erfahrung muss - und das ist hier zu berücksichtigen - der Schutz der Gewohnheiten verlassen werden. Auch erfordert das Erfahrungmachen offene Grenzen zwischen einem bewussten und einem intuitiven, zur affektiven Resonanz fähigen Umgang mit dem Gegenstand der Erfahrung. Hier werden die Bedeutungen von Intuitionen und Phantasien in den Blick zu nehmen sein - Phantasien, denen man sich einerseits öffnen und die man andererseits um der Nachhaltigkeit der Erfahrung willen in (sprachliche) Form bringen muss.

Fassen wir unser Anliegen zusammen: Wir verstehen Erfahrungen vor dem Hintergrund eines pragmatistischen Verständnisses als irritationsbedingte Prozesse, auf deren Basis und in deren Verlauf sich eine intensive Wechselbeziehung zwischen Subjekt und dem Gegenstand der Erfahrung herausbilden kann. Eine Untersuchung der Strukturmomente dieses irritationsbedingten Prozesses verspricht, Konstellationen einer sich sinnhaft entwickelnden Beziehung zu einem Lerngegenstand vor Augen zu führen. Was sich hier - im Zuge eines irritationsbedingten Prozesses und der besagten Wechselbeziehung zwischen Subjekt und Objekt - herausbilden kann, ist Sinn - sowohl in einer hermeneutisch-verstehenden als auch normativ-wertenden Weise (ausführlicher zum Sinnbegriff siehe Combe \& Gebhard 2007; Gebhard 2003). Zu diesem aufscheinenden Sinn gehört auch, dass das Erfahrungsgeschehen in besonders hellsichtigen Momenten in einem lebensgeschichtlichen Zusammenhang vor Augen steht und damit fachliche Lernprozesse biografisch bedeutsam werden können. Eben dies könnten die allenthalben geforderten persönlichkeitswirksamen Lernprozesse sein.

Die Auseinandersetzung mit dem provozierenden Kern des Erfahrungskonzeptes, nämlich der Gedanke eines irritationsbedingten Prozesses, führt uns im Abschnitt $2 \mathrm{zu}$ den Elementen dieses Prozesses und ihrer funktionalen Bedeutung. Dabei wird die besondere Bedeutung und Funktion der Phantasie bei der Verarbeitung und Transformation der Irritation eine zentrale Rolle einnehmen. Im weiteren Verlauf werden wir die Fruchtbarkeit einer Erfahrungstheorie des Lernens für die Gestaltung schulischen Lernens in ersten Umrissen deutlich machen. Deshalb werden wir im Abschnitt 3 prüfen, ob und in welchen Hinsichten es in den Modellen der empirischen, unterrichtsbezogenen LehrLern-Forschung Bezüge zu diesen Elementen des Erfahrungsprozesses gibt. Was auf der theoretischen Ebene ausgeführt und als Erfahrungsbewegung zwischen Subjekt und Gegenstand dargestellt wird, soll in ersten Umrissen auch auf ihre didaktisch-operative 
sowie empirische Anschlussfähigkeit überprüft werden. Im Schlussabschnitt 4 nehmen wir die beiden zentralen „Motoren“ des Erfahrungsprozesses unter dem Titel „Alltagsphantasien" noch einmal auf: Irritation und Phantasie.

\section{Strukturelemente der Erfahrungsbewegung}

Im Folgenden möchten wir die einzelnen Strukturelemente des Erfahrungsprozesses in ihrem Funktionszusammenhang so herausarbeiten, dass der Charakter und die Besonderheit von Erfahrung deutlich wird.

\subsection{Irritation als Beginn des Erfahrungsprozesses}

Dewey (1988, S. 80 ff.) beschreibt den Beginn des Erfahrungsprozesses als ein Geschehen, das aus der Zeit und Kontinuität herausrückt. Die erste Stufe des Erfahrungsprozesses bezeichnet Dewey als Irritation (vgl. hierzu auch English 2005). Die Krisenhaftigkeit dieser Situation drückt sich dadurch aus, dass eingespielte Erwartungen und Routinen versagen. Weiterzumachen wie bisher, ist angesichts dieser Störerfahrung nicht leicht möglich. Die Situation enthält eine „Fremdheitszumutung“ (Combe 2005). Zugleich enthält sie keine Informationen über eine mögliche Reaktion und über eine unmittelbar abrufbare Verknüpfung mit einem bisher erlebten Ereignis. Diese Krise des Gewohnten kann Gegenstand tiefer Beunruhigung sein.

Der Anfang des Erfahrungsprozesses enthält dabei, wie oben schon ausgeführt, einen Moment des „Widerfahrnisses“ (vgl. Bollnow 1968; Waldenfels 2002), also einen Zug des passiven Erleidens. Allerdings verweist der Beginn von Erfahrung auch auf einen aktiven Zug des Sich-Aussetzens an das Neue und Fremde. Hierin steckt die Idee, ja Sehnsucht nach einer Grenzerfahrung, „die das Subjekt von sich selbst losreißt“ (Foucault 1996, S. 46). Wir müssen, so argumentiert Foucault, am besten etwas außerhalb unserer normalen Umwelt und Beschäftigung probieren, Dinge sehen und erleben, denen wir vorher nicht begegnet sind.

Krise verstehen wir als einen Einbruch in einen (gewohnten) Handlungsablauf. Irritation dagegen kann als psychisches Äquivalent dieser Krise der Routine aufgefasst werden. Wenn Erfahrung - jedenfalls in diesem Anfangsstadium - oft als eine „negative" bezeichnet wird, so scheinen hier relativ dramatische, biografisch bedeutsame Einschnitte ins Zentrum der Aufmerksamkeit zu rücken. Aber ein krisenhaftes Initialmoment muss nicht unbedingt ein sensationelles Ereignis sein. Eine subtile Beschreibung dieser „Negativität“" verdanken wir dem phänomenologischen Zugang von Bernhard Waldenfels (2002, S. 99 f.; 2004, S. 65 f.). Er beschreibt, wie „etwas“, eine Gegenständlichkeit also, in einen Routineablauf tritt, Aufmerksamkeit erregt, berührt und stört, ohne dass wir noch sagen oder verstehen könnten, was dieses Etwas ist und bedeutet. Das Geschehen hakt gleichsam im Unbewussten ein. Die damit verbundene Fremdheitszumutung wird nicht immer nur intellektuelles Vergnügen, sondern kann auch Unbehagen bereiten. Man ist gewissermaßen in affektiver und leibnaher Weise alarmiert.

Dennoch ist es alles andere als selbstverständlich, dass ausgehend von diesen Irritationen ein Prozess des Erkundens und Forschens erfolgt. Waldenfels hat auf Sperren 
hingewiesen, die bezüglich der Entscheidung bestehen, ob man sich der irritierenden Situation überhaupt aussetzen soll. Er ermöglicht uns in seinen Arbeiten Einblicke in die differenzierten Stufungen und Übergänge zwischen Akzeptanz und Nicht-Akzeptanz der irritierenden Situation. Er wählt für diesen Spannungszustand eine topologisch-räumliche Metapher: Eine Schwelle sei zu überqueren (Waldenfels 2004, S. 22); diese Schwellenüberschreitung zeigt eine körperlich-räumliche Entfernung vom Vertrauten an und sie führt ins Ungewisse: Man weiß selten, was einen jenseits der Schwelle erwartet und wie die Sache ausgeht. Der Irritation standzuhalten heißt auch, sich von Komfort und Beharrungskräften zu lösen.

Ein die Person erreichendes Lernen hat also keinesfalls nur harmonische Ausgangskonstellationen zur Voraussetzung. Es muss jetzt bedacht werden, was denn dazu ermutigen kann, sich auf Wege in fremde Sinnwelten zu begeben. Wie können, so lautet die Frage, die Irritationen in konstruktive Lernprozesse transformiert werden und geradezu als Motor für Lernprozesse und als „fruchtbarer Moment im Bildungsprozess“ (Copei 1969) fungieren? Der krisenhafte, irritierende Zustand muss also noch weiter ausgelotet werden.

\subsection{Die Krisenkonstellation als fruchtbarer Moment}

Im Folgenden wird es um die Frage gehen, ob und unter welchen Bedingungen man sich der irritierenden Situation überhaupt aussetzen will. Wir halten die Klärung dieser Frage - auch vor dem Hintergrund unserer Fallrekonstruktionen (Combe 2005; Combe \& Gebhard 2007) - für zentral. Die Psychoanalyse spricht hier von Widerstand oder von Abwehroperationen (Bittner 1998, S. 166 f.), also von verschiedenen defensiven Maßnahmen, um sich den Anspruch der Situation vom Leibe zu halten. Die irritierende Situation kann Unlust hervorrufen, sich bisweilen auch als kränkend erweisen und berührt uns in unserer leibnahen Affektkonstitution. Das kann dazu führen, dass solche Situationen gemieden werden oder - was weitaus häufiger der Fall sein wird -, dass die mentale Beschäftigung damit gewissermaßen zurückgewiesen wird. Vermieden wird also nicht die Irritation (das geht in der Regel nämlich nicht), sondern die Beschäftigung damit, also die Reflexion derselben. Eben diese Beschäftigung wäre jedoch - und das ist eine unserer zentralen Thesen - eine Bedingung dafür, dass das Potenzial der Krise, der Irritation zu fruchtbareren Momenten werden kann. Hier taucht also schon in der Impulszone des Erfahrungsprozesses das Problem auf, ob man bereit ist, sich überhaupt einer irritierenden Situation zu öffnen, einer krisenhaften Situation, die nur fruchtbar werden kann, wenn bewährte Vorstellungen überschritten und transformiert werden.

Das Problem der Akzeptanz der irritierenden Situation wird noch komplexer, bedenkt man zusätzlich den Unterschied zwischen Krisen und Irritationen in der Handlungsumwelt einerseits und Krisen in dem Bereich einer symbolisch ausgeformten und anzueignenden „Welt des Geistes“ andererseits. Zunächst haben „geistige“ Krisen durchaus den Geruch des Überflüssigen an sich, wie Hans Blumenberg (1987) anmerkt. Und Ulrich Oevermann (2004) weist zu Recht darauf hin, dass man auf Krisen, die durch Katastrophen oder Entscheidungssituationen gekennzeichnet sind, reagieren muss, während eine geistige Krise einen auch selbsterzeugten Charakter hat. Mit anderen Worten: Die Krise im Bereich der geistigen Weltbegegnung ist eine Krise mit vergleichsweise gerin- 
gem Handlungsdruck. Sie ist im Prinzip vermeidbar, wird aber durch das Subjekt in oft unermüdlichen Anläufen selbst herbeigeführt. Bei diesem neugierigen Verfolgen von müßigen Fragen muss ein motivierendes Moment hinzukommen, damit man sich auf den Anspruch und die Herausforderung einer Situation einlässt. Was führt und motiviert, so lautet die Folgefrage, also zu dieser müßiggängerischen Problematisierung, die wir aus dem Bereich der ästhetischen Erfahrung, aus dem Umgang mit Kunst, Musik und Literatur kennen? Was führt zum Sich-Einlassen auf die irritierende Problemsituation, zur Öffnung gegenüber dem Gegenstand, die schließlich eine Erfahrungsbewegung dadurch bewirkt, dass man diesem immer neue Seiten abgewinnt?

Ein konstruktiver und nicht widerständiger Umgang mit besagter Irritation ist, so lautet unsere These, die Öffnung eines Vorstellungs- und Phantasieraumes. An dieser Wegscheide des Erfahrungsprozesses, wo es um die Akzeptanz von Fremdheit geht, erweist sich der Umgang mit Phantasieprozessen als zentral dafür, ob und wie Irritationen in konstruktive Lernprozesse transformiert werden können. Diese Öffnung eines Phantasieund Vorstellungsraumes treibt den Erfahrungsprozess über das Verharren im Widerständigen hinaus.

\subsection{Die Öffnung eines Vorstellungs- und Phantasieraumes - Phantasie als Motor der Erfahrungsbewegung zwischen Subjekt und Lerngegenstand}

Auf den Begriff der Phantasie im Hinblick auf Lernprozesse zu rekurrieren, ist im Rahmen der bisherigen didaktischen, aber auch der lerntheoretischen Diskussion nicht selbstverständlich. Dies liegt nicht zuletzt an einer gewissen Unschärfe des Phantasiebegriffs. Es geht uns in diesem Abschnitt vor allem um die Begründung der These, dass und inwiefern die Öffnung eines Vorstellungs- und Phantasieraumes der entscheidende Schritt für die Produktivität der Erfahrungsbewegung zwischen Ich und Gegenstand ist. Dieser Schritt führt über die Irritation und einen möglichen Widerstand hinaus und macht verständlich, warum man den Anspruch geistiger Krisen auf sich nimmt.

Einige kurze Bemerkungen zum Begriff der Phantasie seien vorangestellt: Innere Bilder, Vorstellungen und ihre kreative Version, nämlich Phantasien, sind auf abwesende Objekte gerichtet, während die Wahrnehmung sich auf die ,phänomenale Repräsentanz“ (Prinz 1983, S. 379) anwesender Gegenstände bezieht, mit denen man im handelnden Umgang Kontakt hat. Zur sinnlichen Wahrnehmung gehört die Anwesenheit der Objekte, zur sinnlichen Vorstellung jedoch nicht. Begriffe wie Vorstellung, innere Bilder, Phantasien und Imaginationen liegen - trotz ihrer unterschiedlichen theoretischen Herkunft - insofern auf einer Linie, als für sie charakteristisch ist, dass ihre Objekte ,anschaulichabwesend" sind (Sartre 1971, S. 57).

Über die Bedeutung der Phantasie ist in der Philosophie in erkenntnistheoretischer, ethischer, ästhetischer und religiöser Hinsicht vielfach nachgedacht worden (vgl. die Übersicht bei Ritter 1971). Das Wort wurde im 14. Jahrhundert in das Deutsche eingeführt und wird von Anfang an ambivalent gebraucht, denn immer wieder ist auch Misstrauen gegenüber der Phantasie - sozusagen als „Phantasterei“ - zum Ausdruck gebracht worden.

Diese Kritik berührt auch die Frage, was konstruktive, zukunftsgewandte Phantasien von lähmenden Phantastereien unterscheidet (vgl. Oettingen 1997; Oettingen, Pak \& 
Schnetter 2001). Andere Ausdrücke sind in das Wortfeld eingeführt worden: Einbildungskraft, Imagination, Vorstellung, Intuition - um nur die Wichtigsten zu nennen. Und immer wieder fungierte Phantasie als Gegensatz zum rationalen Denken.

Im Hinblick auf fachliche Lernprozesse ist ein Gedanke bedeutsam, für den aufgrund der Phänomenologie des Erfahrungsprozesses viel spricht: Die Phantasie ist das organisierende Zentrum eines Wechselverkehrs zwischen Ich und Gegenstandswelt, ein Übergangsraum, in dem der Mensch sich quasi oszillierend zwischen seinen innerseelischen Prozessen und den materiellen Gegebenheiten der äußeren Welt, in der er sich gerade befindet und mit der er sich befasst, hin und her bewegt (vgl. hier auch Winnicott 1995; Schäfer 1986, 1995; Mollenhauer 1996).

Betrachten wir nun im Einzelnen, welche Rolle Phantasien im Zuge der Auflösung und Transformation von Widerständen bieten. Im Falle des Widerstands kommt es statt zu einer Öffnung gegenüber dem Gegenstand zu einer Verengung. Der irritationsbedingte Prozess wäre zu Ende, bevor er in produktiver Weise begonnen hat; anders im Falle der Eröffnung eines Vorstellungs- und Phantasieraumes. Wir sehen hier vor allem zwei theoretische Varianten:

Die erste Variante des Phantasiebegriffs lässt sich von der psychoanalytischen Abwehrtheorie herleiten. Die Annahme ist, dass ein Großteil der Abwehrvorgänge dazu dient, vor Kränkungen, Verletzungen und Einbrüchen des Selbstgefühls zu schützen, wie umgekehrt ein stabiles Selbstwertgefühl die Notwendigkeit von Abwehroperationen herabsetzen dürfte (Hoffmann 1987, S. 34). Die Abwehroperationen werden hierbei in einen narzisstischen Zusammenhang und in den Kontext der Selbstpsychologie gestellt (vgl. zu Erweiterungen der Psychoanalyse in Richtung Selbstpsychologie, Bittner 1998). Angewandt auf das Problem des Sich-Einlassens auf eine irritierende Ausgangssituation von Erfahrungsprozessen bedeutet dies, dass eine Phantasie des Gelingens und damit verbunden eine Vorfreude auf ein gelungenes Werk eine konstruktive Wendung herbeiführen könnte. Man könnte hier geradezu von einer Utopie des Gelingens sprechen. Es genügt also nicht, nur den irritierenden Anfang eines Erfahrungsprozesses zu betrachten, sondern es gilt, diesen auch von seinem - wenn auch fragilen - Ende her in Augenschein zu nehmen. Hierbei ist die Krise also nur ein Durchgangsstadium eines Prozesses, an dessen Ende ein Zustand des Gelingens steht - ein zwar flüchtiges Glück, das jedoch das Verlangen nach Wiederholung und Dauer wecken kann. Unsere Überlegung ist also, dass vom Überwinden der Krise eine tiefe Bestätigung ausgeht. Am - natürlich vorläufigen - Ende des Prozesses steht ein erweitertes Ich: Man sieht etwas, was man bisher nie so gesehen hat. Dass ein stabiles Selbstwertgefühl, Zuversicht und Selbstvertrauen die Notwendigkeit von defensiven Abwehrmaßnahmen herabsetzen können und für eine produktive Krisenlösung unerlässlich sind, davon spricht schon Nietzsche, indem er auf den narzisstischen Motor der Phantasie verweist. In einem ,genialischen Vorgefühl“ des Gelingens liegt, so Nietzsche, ,eine treibende Kraft und die Hoffnung auf zukünftige Fruchtbarkeit“ (Nietzsche 1966, Bd. III, S. 362). Aber Nietzsche lässt keine Zweifel daran, dass in dieser Form der projektiven Imagination nur vorweggenommen wird, was später in Gestalt der realen Begegnungen und Anforderungen noch durchlebt werden und entsprechende Wirklichkeitsraster finden muss. Ansonsten spräche man von Phantasterei. Dem Prozess der Phantasierealisierung nähert sich nun das pragmatistische Konzept. 
Diese zweite Variante des produktiven Umgangs mit Zuständen der Irritation lässt sich vom Pragmatismus her entfalten und nimmt die Bedeutung der Eröffnung eines Vorstellungs- und Phantasieraumes in den Blick. Oevermann (1991, S. 267 ff.) hat im Anschluss an George Herbert Mead herausgearbeitet, inwiefern Phantasien und innere Bilder als Medium einer gedankenexperimentellen Abarbeitung von Problemkonstellationen und Problemlösungen fungieren. Mittels der Phantasie und korrespondierenden inneren Bildern findet eine intensive Austauschbewegung zwischen Ich und der Sache statt; es kommt zu Konstruktions- und Rekonstruktionsbewegungen, bei denen die inneren Bilder gleichsam als „Text“ anzusprechen sind. Phantasien sind somit auch Ausdrucksgestalten, in denen die Erfahrungsbewegung zwischen Ich und der Sache außerhalb ihres gegenwärtigen Vollzugs festgehalten und kommuniziert werden kann. Diese von der Sozialphilosophie Meads herkommende Variante der Funktionsweise der Phantasie bedeutet letztlich Sinnarbeit - ein anspruchsvoller Prozess der Konstruktion und Rekonstruktion einer Problemlage und ihrer möglichen Lösungen. Diese sind das Werk einer von Vorerfahrungen gespeisten Arbeit mit inneren Bildern und Phantasien, die dann je neu zu verlängern sind im Sinne von hypothetischen Entwürfen des Zukünftigen. Interessanterweise lehnen sich die psychologischen Forschungsaktivitäten über die mentale Konstruktion von Vorstellungsbildern, selbst wenn sie in einer anderen Terminologie gefasst sind, der funktionalen Konzeption von Phantasie als „Text“ an: „Diese Vorstellungstätigkeit ermöglicht uns, ,vor unserem geistigen Auge' statische und dynamische Bilder von realen oder erdachten Szenen und Vorgängen entstehen zu lassen, die dann vor diesem inneren Auge betrachtet und analysiert werden können“" (Bannert \& Schnotz 2006, S. 73). Die Ingangsetzung einer auf Herausforderung und zugleich Lösung bezogenen Phantasie geschieht allerdings nicht von allein. Phantasien brauchen ,,ihr“ Setting: zum einen Handlungsentlastungen und Rückzugsräume und zum anderen innere Bündelung der Konzentration. Man muss Ruhe haben, aber auch eine gewisse Konfliktspannung in sich tragen, damit gleichschwebend, mußevoll, fast hinter dem Rücken des Ichs Eindrücke, Erinnerungen und Phantasien produziert und umstrukturiert werden können, die dann als neuer Vorstellungszusammenhang wieder ins Bewusstsein eintreten. Diese Phantasieszenarien sind Ideenkeime einer möglichen Problemlösung. Sie organisieren sich um, lassen sich wie Texte zu neuen Zusammenhängen fügen, bis eine ,gestaltrichtige“, d.h. eine lösungsversprechende Zuordnung der inneren Bilder zu objektiven Krisen- und Problemkonstellationen möglich ist.

Die Phase der Produktion innerer Bilder hat dabei einen monologischen, aber zugleich einen auf andere Subjekte gerichteten Zug, die damit quasi zu Ko-Konstrukteuren werden. Wir streben also auch nach sozialer und sachhaltiger Validierung unserer subjektiven Projektionen und Symbolisierungen. Wie sieht mein Problem in seiner allgemeinen Verfasstheit aus? Was machen andere in dieser Lage? In der Phase der Irritation und Krise ist jedoch jeder ein Stück weit mit sich allein. Bei dem Bedürfnis, Handlungs- und Interpretationsmöglichkeiten in einem sozialen Austausch zu überprüfen, geht es hingegen um die Anschlussfähigkeit unseres inneren Dialoges. Die Herstellung von Anschlussfähigkeit ist dabei in hohem Maße eine Leistung von Sprache - also der Eröffnung eines sprachlich-dialogischen Artikulationsraumes. Für die nachhaltige Bewältigung eines Problems ist es wichtig, dieses und die damit verknüpften Phantasieszenarien in Worte fassen zu können. Verfolgen wir also die Dramaturgie des Erfahrungsprozesses weiter. 
2.4 Dem Neuen Begriffe geben: Die Öffnung eines sprachlichen Artikulationsraumes durch Metaphorik

Kennzeichen der sprachlichen Artikulationsebene von Erfahrungen ist die Suche nach einer Sprache, in der Erfahrungen, Wünsche, Phantasien und Emotionen artikuliert werden können, die noch keinen (sprachlichen) Ausdruck finden konnten. Zudem nimmt die Sprache Bezug nicht nur zur inneren Phantasieebene, sondern auch zur äußeren Realität. Dafür haben wir mit dem Begriffspaar der Subjektivierung und Objektivierung ein didaktisches Modell entwickelt (Combe \& Gebhard 2007; Gebhard 2005).

Für den weiteren Erfahrungsprozess und dessen produktive Transformation bedeutet dies, dass jetzt dem Neuen Begriffe gegeben werden müssen. Die der Erfahrung entgegenkommende Form der Mitteilung kann die Erzählung sein, an der, wie Walter Benjamin in seinem 1936 verfassten Aufsatz „Der Erzähler“ schreibt, „die Spur des Erzählenden wie die Spur der Töpferhand an der Tonschale [haftet]“ (Benjamin 1977, S. 447). Die Bedeutung der Sprachlichkeit hervorzuheben heißt indes nicht, dass Prozesse der Erfahrungsverarbeitung stets in Eindeutigkeit und definitivem Abschluss enden. Die „Wahrheit" kann in diesem Zusammenhang oft nicht mehr und nicht weniger sein, als ,ein bewegliches Heer von Metaphern“ (Nietzsche), weil gerade im Fluss der Bilder Perspektiven auf eine neue Sicht der Dinge gedanklich erprobt und artikuliert werden können. Wiederum ist hier auf die für die sprachliche Artikulation wegbereitende Funktion der Phantasie hinzuweisen. Sofern wir von Risiken und Zwängen der Praxis entlastet sind, können wir das Geländer loslassen, das uns daran hindert, zur Symbolisierung (noch) „phantastischer“ Möglichkeiten weiter vorzudringen. Begriffe rücken einen Erfahrungszusammenhang in eine allgemein verbindliche Sprachregelung und in ein allgemeines Bedeutungsuniversum ein. In diesem Sinne sind sie, wie Bertolt Brecht sagt, „Griffe“. Aber die begriffliche Artikulation der Erfahrung fixiert auch, setzt fest, abstrahiert vom Fluss der Erscheinungen.

\subsection{Zwischenbilanz}

Wir haben Erfahrung als irritationsbedingten Prozess dargestellt, der schließlich seinen Platz in einer bedeutungsvollen Lerngeschichte finden kann. So entstehen „Geschichten“, die man erzählen kann. Dass dieses Erfahrungsgeschehen schließlich in eine sprachliche Welt eingerückt wird, bedeutet nicht, dass dieses Geschehen notwendig der rationalen Kontrolle unterliegt. Das wäre eine allzu intellektualistische Variante des Erfahrungsprozesses. Zwar ist die spezifische menschliche Möglichkeit einer sich durch Sprache erweiternden Erfahrung hervorzuheben; ein Nachdenken über eine Erfahrung kann durchaus $\mathrm{zu}$ einem differenzierten Erleben zukünftiger Erfahrung führen. Aber ein Gutteil der Erfahrung entzieht sich dem Bewusstsein und der Kontrolle. Im Falle einer Irritation und Störerfahrung ist mit einem Prozess zu rechnen, der sich auf der Ebene körperlich-affektiver Resonanzen anbahnt und zunächst einmal zu spontanen Passungsversuchen oder auch Widerstand führt. Wir haben gefragt, ob solche Irritationen oder krisenhaften Konstellationen auch fruchtbar werden können. Als entscheidend für dieses Fruchtbarwerden haben wir die Phantasie als einen entscheidenden Motor von Lernprozessen herausgestellt. Menschen haben die Freiheit der konstruktiven Phantasie. Die Phantasie ist durch ihre 
vermittelnde Repräsentationsqualität das vorantreibende Element, das Transformationen des Verhältnisses zwischen Subjekt und Sache bewirkt. Die Funktionsweise der Phantasie erschließt sich dabei weniger in Form einer linearen, nur nach vorne gerichteten Bewegung. Ihre Bewegungsform ist vielmehr die einer zyklischen Entfaltung - in Rück- und Vorgriffen, Entwürfen und deren Evaluation. Dieser „rückgreifende Vorgriff“ (Combe 1992) besteht also in durch bisherige Erfahrungen vorgebildeten Entwürfen und einer analog strukturierten Bewegung der Rekonstruktion und Bewertung des Entworfenen.

Im weiteren Verlauf soll es darum gehen, das Potenzial einer Erfahrungstheorie des Lernens für die Gestaltung schulischen Lernens wenigstens in ersten Umrissen deutlich zu machen. Zugleich soll skizziert werden, dass es in den Modellen der empirischen, unterrichtsbezogenen Lehr-Lern-Forschung Bezüge zur Prozessstruktur von Erfahrung gibt. Dieser hier freilich nur kurz und exemplarisch zu berührende Zugang bedeutet, dass didaktische und empirische Ansätze daraufhin befragt werden, ob sie zentrale Strukturelemente des Erfahrungsprozesses abbilden:

- Die Irritation als subjektive Eröffnungsbedingung eines Erfahrungsprozesses

- Spontanreaktionen und Formen der Resonanz, die eine Öffnung gegenüber dem Gegenstand ermöglichen oder verhindern

- Das Aufsteigenlassen von Bildern und Phantasien narzisstischer, konstruktiver und rekonstruktiver Provenienz: Entwürfe ins Offene und deren „Evaluation“

- Reflektierende Besinnung und das Bemühen um einen narrativen, figürlichen und begrifflichen Ausdruck des Neuen

Wir möchten bei der Inbezugsetzung zur Didaktik und zur empirischen Lehr-Lern-Forschung folgendermaßen verfahren: Bezüge zur Forschung und didaktischen Praxis sollen sich zunächst auf Analogien zu den Vollzugs- und Ausdrucksformen von Erfahrungsprozessen und deren funktionale Bedeutung beziehen, also auf Irritation, Lernwiderstand, Phantasie und schließlich Metaphorisierung und Verbegrifflichung von Erfahrung. Diese Kategorien der Organisation von Erfahrung werden in didaktischer Beleuchtung zugleich konkretisiert. Es wird sich zeigen, dass ein Problem darin liegt, die einzelnen Merkmale eines Erfahrungsprozesses als isolierte Bausteine zu betrachten. Ohne einen erlebten und auch netzwerkartig gedachten Zusammenhang zwischen allen Komponenten kann sich - so unsere Vermutung - ihre Lernwirksamkeit nicht entfalten. Ein solcher Zusammenhang stellt sich schließlich in der Projektform des Unterrichts (Abschnitt 3.5) sowie in der unterrichtlichen Arbeit mit Alltagsphantasien (Abschnitt 3.6) her.

\section{Zur didaktischen und empirischen Anschlussfähigkeit des Erfahrungskonzepts}

\section{1 „Aufgabenkultur“ und die Annäherungsarbeit an den Lerngegenstand}

Wir werden nun im Bereich der empirischen Lern- und Unterrichtsforschung sowie der Didaktik nach Ansätzen Ausschau halten, die eine gewisse prozedurale Komplexität beinhalten und die von der Auflösung einer irritierenden, herausfordernden und krisenhaften Anfangskonstellation ausgehen. Es lässt sich hier auf Unterrichtsmuster zurückgreifen, wie sie in Videostudien rekonstruiert worden sind (Baumert et al. 1997; Stiegler 
\& Hiebert 1999; Hiebert et al. 2003). Hier hat sich vor allem im so genannten japanischen Muster, einem aus mathematikdidaktischer Sicht guten Unterricht, eine zeitintensive Auseinandersetzung mit wenigen, aber kognitiv anspruchsvollen, komplexen Aufgaben als ein organisierendes Zentrum eines solchen Unterrichts herausgestellt. Von dieser kognitiven Aktivierung, etwa durch eine herausfordernde Aufgabenkultur, lässt sich ein Zusammenhang zum Gedanken der Irritation bzw. Krise als Motor von Lernprozessen herstellen. Hiervon kann das Fragen ausgehen: Es ist durch Spannungen motiviert und mündet in Suchmuster für neue Erfahrungen.

Parallel zu dieser „Grunddimension“ der kognitiven Aktivierung (Klieme 2006, S. 768 f.) hat sich das Aufgabenverständnis im Bereich der Didaktik ausdifferenziert bzw. es hat eine Revision der Aufgabenkultur eingesetzt (siehe ausführlich Eikenbusch 2008). Worum es hierbei in Analogie zum irritierenden Beginn von Erfahrungsprozessen, auch im Hinblick auf die Aufgabenkultur, geht, ist eine Annäherungsarbeit an den Lerngegenstand, die nicht ,die geläuterten Objekte der Wissenschaft“ (Dewey 2007, S. 37) präsentiert. Lerngegenstände sind den Lernenden nicht nur als äußerliches Gegenüber gegeben, sondern auch im Modus von Umgangserfahrungen. „Denn Dinge sind in viel höherem Maße Objekte, die behandelt, genutzt, auf die eingewirkt, mit denen gewirkt werden soll, die genossen und ertragen werden müssen [...] Sie sind Dinge, die man hat, bevor sie Dinge sind, die man erkennt“" (Dewey 2007, S. 37).

\subsection{Irritationen als Riss zwischen den Wissensformen: Alltagsvorstellungen und fachsprachliche Deutung}

Eine fast alltägliche Form der Irritation beim schulischen Lernen, die die Notwendigkeit einer „Annäherungsarbeit“ bedingt, ist die Kluft zwischen Alltagsvorstellungen und fachlichen Wissenssystemen. Irritationen spielen also eine Rolle beim Übergang von lebensweltlichem zu wissenschaftlichem Wissen (Meyer-Drawe 1996). Was an wissenschaftlichem Wissen schließlich curricular ,archiviert“ wird, entwickelt sich mit einer eigenen Selbstbezüglichkeit. Die vor allem naturwissenschaftsdidaktische Alltagsvorstellungsforschung hat diesen schwierigen und auch oft konfliktreichen Übergang unter dem Stichwort „conceptual change“ gut erforscht (Duit 1996, 2006; Reinfried 2007). Dennoch liegt noch ein offenes Forschungsfeld vor. Die Übertragbarkeit der Befunde etwa auf sprachliche Fächer kann als noch nicht ausreichend geklärt gelten (Klieme 2006, S. 770 ff.).

Das Subjekt wird bei der Konfrontation mit neuem (wissenschaftlichen) Wissen in seinen bewährten Alltagsvorstellungen gewissermaßen gestört. Dass dies zumindest auch eine krisenhafte Situation ist, haben wir an anderer Stelle ausführlich herausgearbeitet (Combe \& Gebhard 2007). Dass Lernende bei der ersten Annäherung an einen fachlichen Gegenstand ein Fremdheitsgefühl haben, d.h. dass ein Abstand zwischen Person und Sache zu beobachten ist, ist aus der Sicht der Naturwissenschaftsdidaktik charakteristisch für den Anfang eines Lernprozesses. Entscheidend ist dabei, wie mit diesem Abstand durch die Lehrkraft umgegangen wird. Es ist nicht sinnvoll, den Abstand so schnell wie möglich überwinden zu wollen. Vielmehr gilt es, diesen zu einem Spannungsfeld zu machen, aus dem Lernprozesse ihre Dynamik beziehen. So wird ein ,kognitiver Konflikt" - genauer: eine konzeptuell bestimmte Krise - geradezu dadurch erzeugt, dass 
verschiedene Sichtweisen, die alltäglichen und die wissenschaftlichen, einander gegenübergestellt werden (Duit 1993, 1996, 2006; Kattmann \& Gropengießer 1996). Das Ziel ist insofern nicht die Eliminierung der alltäglichen Vorstellungen, sondern die Fähigkeit der „Zweisprachigkeit“ (Combe \& Gebhard 2007; Gebhard 2005), die es gestattet, zwischen beiden (Sprach-)Versionen spielerisch und phantasievoll hin- und herzugehen. Auch mit dieser ,konzeptuellen Krise“, wie wir sie mit Blick auf die Kluft zwischen den Wissensformen nennen wollen, haben wir nur den Ausgangspunkt einer Erfahrungsbewegung bezeichnet. Aber unter gegenwärtigen schulischen Bedingungen ist nicht sicher, ob dieser Erfahrungsprozess nicht im Widerstand stecken bleibt, bevor er überhaupt in produktiv-praktischer Weise in Gang kommt.

\subsection{Widerstände und schulische Bedingungen: Imagery-Strategien und Fehlerfreundlichkeit}

Wir haben die Phantasie charakterisiert als das organisierende Zentrum eines Wechselverkehrs zwischen Ich und Gegenstandswelt, als einen Übergangsraum (nach Winnicott 1995; Schäfer 1986, 1995). Entschlossenheit und Wille reichen nicht, um produktive Phantasien „herbeizuführen“. Die Eröffnung eines Phantasieraumes erfordert eine Öffnung gegenüber den Quellen jenseits bewusster Überlegung und Zwecksetzung. Ein Beispiel für das hier entstehende Zusammenspiel zwischen innerer und äußerer Welt ist das Spiel des Kindes: Die Phantasie, aus der inneren Welt kommend, holt zugleich die äußere Welt ins Ich hinein (vgl. Winnicott 1995; Schäfer 1986).

Die daraus ableitbare Frage an das didaktische Setting ist, ob es entsprechende Räume für das subjektive Andocken von Phantasien an den Lerngegenstand gibt - auch in ihrer Funktion zur Überwindung von Lernwiderständen. Dazu kommt, dass sich solche Gestaltbildungen einer Problemsituation und ihrer möglichen Lösungen auch am Widerstand gegenüber einer Sache erst entzünden. Der besondere Beitrag der funktionalen Phantasie zur Krisenlösung liegt in ihrer Funktion eines Mediums zur Konstruktion und Rekonstruktion, mittels derer die Transformationen von der Problemstruktur zur Lösungsstruktur vorangetrieben werden können. Zunächst wird dieser Diskussionsstrang in der Pädagogischen Psychologie unter den Stichworten der Konstruktion und Veränderung ,mentaler Modelle“ (vgl. etwa zur Übersicht Seel 2000, S. 253 ff.) sowie unter dem Stichwort „Vorstellungsbilder“ und „Imagery-Strategien“ geführt. „Mentale Modelle dienen“, so Seel, „der Simulation ,möglicher Welten“ und ihrer möglichen Transformationen“" (Seel 2000, S. 256). In diesem Zusammenhang liegen seitens der Psychologie zahlreiche Untersuchungen darüber vor, dass sich die Durchlässigkeit zwischen den Repräsentationsformen sowie eine Mehrfachkodierung, etwa im ikonischen und sprachlichen Format, positiv auf das Lernergebnis auswirken können (Bannert \& Schnotz 2006). Dennoch werden auch nach dem Urteil von Bannert und Schnotz Imagery-Strategien, etwa beim Lesen oder der Textverarbeitung, allzu sparsam in der Schule eingesetzt (vgl. auch Gudjons 2003).

Das hier angesprochene unterrichtliche Defizit hinsichtlich der Möglichkeiten der Ausgestaltung eines Vorstellungs- und Phantasieraumes zur Herstellung einer breiten Berührungszone zwischen Innen- und Außenwelt eines Lerners lässt sich noch in einer etwas anderen Weise beleuchten, nämlich von den Bedingungen aus, die den Umgang mit Phantasien begünstigen. Wir meinen die Fehlerfreundlichkeit des Unterrichts: Ein „diskursi- 
ver Umgang mit Fehlern“ (Klieme 2006, S. 770) kann zunächst als „Basisdimension“ bezeichnet werden, die eine korrespondierende Bedingung zur kognitiven Aktivierung im Unterricht darstellt. Das Problem liegt darin, dass Schüler, wenn sie sich offen und vertrauensvoll mit Lernproblemen, Unwissen, Nichtverstandenem und mit immer wiederkehrenden Problemen an ihre Lehrer wenden, damit ein Risiko eingehen (empirisch: Hascher 2005). Wenn hier Sanktionen, Beschämung und Bloßstellung drohen, wird eine reflektiert-spielerische Selbstdistanz, ein suchend-erprobender Umgang mit der Irritation kaum zu erwarten sein. In diesem Zusammenhang ist die Lernforschung wie auch die Didaktik für Formen des Feedbacks im Unterricht sensibel geworden (Helmke 2003; Bastian, Combe \& Langer 2003). Mit der Fehlerfreundlichkeit ist eine wichtige Bedingung für die positive Nutzung von Phantasieprozessen zur Überwindung von Widerständen bezeichnet. Sichtbar wird außerdem, dass die mit dem Erfahrungslernen verbundenen Erfahrungsstrukturen eine Raum gebende Haltung erfordern, die, in der Terminologie der Lernpsychologie formuliert, sowohl eine kognitive Aktivierung (qua Irritation) als auch eine mentale Modellierung des Lernproblems (qua Phantasie) ermöglichen (vgl. etwa Seel 2000, S. 253 ff.). So können sich Problemlösungsprozesse auch umwegig und in intuitiven oder experimentellen Formen der Annäherung anbahnen, bevor sie eine sprachliche oder sonstige gegenständliche Gestalt gewinnen. Unser Verständnis einer Erfahrungstheorie des Lernens gibt jedenfalls auch der experimentellen Verknüpfung von Handlungsweisen und damit einer durchaus präreflexiven Umgangserfahrung Raum.

\subsection{Erfahrungsgenerierende Ordnungen des Sprechens im Unterricht: Sprachräume}

Unter dem Blickwinkel der Bemühung um Ausdruck und Artikulation von Erfahrung steht das Gesprächskonzept des Unterrichts zur Disposition (zur Sprache im Unterricht: Lüders 2003). Hier geht es um den Bereich der durch Sprache und Bedeutung erweiterten, ja generierten Erfahrungsmöglichkeiten. Nach den Videoanalysen der TIMMSForscher können wir von relativ eingefahrenen Ordnungen des Sprechens ausgehen, die eine schnelle, kleinschrittige Vereindeutigung von Sachverhalten anzielen (vgl. hierzu im Einzelnen auch Rumpf 2004). Die Frage ist nun, ob und inwiefern Erfahrungen im unterrichtlichen Dialog gemacht werden können bzw. ob das stattfindet, was man Erfahrungsbildung nennen kann. Die Strukturen einer erfahrungsbezogenen oder auch erfahrungsgenerierenden Gesprächssituation, in der die Beteiligten füreinander wohl Geburtshelfer sein müssten, hat Käte Meyer-Drawe (1996, S. 95) folgendermaßen beschrieben: „Darum kann es geschehen, daß ich erst im Sagen und Handeln erfahre, was ich wußte, daß erst die Fragen eines Anderen meine Antwortmöglichkeiten hervorbringen und nicht nur abrufen“. Der andere Erfahrungshintergrund meines Gegenübers, seine andere Sicht auf die Dinge fordert mich dazu heraus, meine eigene Position zu überdenken. Ein solch allmähliches Verfertigen von Gedanken und Entstehenlassen von Einsichten im Gespräch könnte aufgrund der empirischen Befundlage durchaus auch auf das Gespräch zwischen den Schülern bezogen werden. Erfahrungen werden dann gemacht, wenn Lernende in sprachlichen Aushandlungsprozessen gemeinsam Wissen konstruieren können (Rehm 2003, S. 206 ff., S. 250 ff.). 
Im Moment konzentriert sich die Diskussion um das Unterrichtsgespräch auf die Dimension der Frage. Fragen und krisenhaftes Fragenkönnen wird in den Untersuchungen der Gesprächssituationen des Unterrichts als entscheidende, Erfahrungen einleitende Dimension beschrieben (vgl. die Übersicht von Neber 2004, 2006; Niegemann 2004). Mit Fragen bahnen wir uns Wege. Nicht selten beginnt darauf bezogen bzw. aufbauend eine Form erschließender Imagination. Das kann bedeuten, dass das Subjekt sich der Irritation zuwendet und damit ein Prozess der experimentellen Problemlösung (im Medium der Phantasie) in Gang kommt. Diese hier wiederum nur skizzierte Genese eines sachorientierten Erfahrungszusammenhangs hat Gadamer (1960, S. 375) folgendermaßen auf den Punkt gebracht: „Einen Text verstehen heißt, die Frage verstehen, auf die der Text eine Antwort ist.“

\subsection{Erfahrungstheorie und Projektmethode}

Indem wir die Strukturstellen des Erfahrungsprozesses in Verbindung zu einzelnen empirischen und didaktischen Annahmen und Befunden gebracht haben, sind Hinweise für die Gestaltung von Lernumgebungen möglich, die für eine Erfahrungsbildung und Erfahrungsverarbeitung günstig sind. Allerdings ist damit noch kein Setting in Sicht, das ein „ganzheitliches“, netzwerkartiges Zusammenwirken der Strukturelemente von Erfahrung $\mathrm{zu}$ einer bedeutungsgeladenen Handlungs, Erlebnis- und Reflektionseinheit verkörpert. Denn eine Erfahrungsbewegung besteht in unserem Verständnis aus Strukturelementen, deren Reihenfolge zwar nicht stufenartig und starr festgelegt ist, die aber aufeinander verweisen, sich wechselseitig aufrufen, Variationen ermöglichen und schließlich in der Art eines „chunking“ als biografisch bedeutungsvolle Einheiten realisiert und abgespeichert werden.

In der angelsächsischen Diskussion existieren Lehrmodelle, deren Kennzeichen den Prozessvariablen von Erfahrung zu entsprechen scheinen. Genannt seien die Instruktionsmodelle der Cognitive Apprenticeship, des Anchored Instruction (Verankerung der Lerninhalte in anregenden Episoden) sowie die Theorie der Cognitive Flexibility (Cognition and Technology Group at Vanderbilt 1997). Die Merkmale idealer Lernumgebungen, die in der Konzeption des Cognitive Apprenticeship gebündelt wurden (Collins, Brown \& Newman 1989), lassen sich in Analogie zu Strukturelementen von Erfahrungsprozessen beschreiben. Entsprechend komplex muss die Aufgabenstellung gewählt werden. Materialien, die zum Experimentieren, zum Explorieren und zur Hypothesenbildung einladen, sind ebenso relevant wie Artikulation, kommunikative Klärung, Revision und Weiterentwicklung von Lösungsoptionen. Zu kritisieren ist allerdings, dass nur wenige Untersuchungen das meist spiralförmige Ineinanderspielen solcher Strukturelemente im Sinne einer abgrenzbaren bedeutsamen Einheit von Erleben, Handeln und Reflexion berücksichtigt haben (Casey 1996). Das aber wäre erforderlich, wenn von Erfahrung gesprochen wird. Hier ergibt sich sowohl ein theoretisches als auch ein methodisches Problem.

Das Schicksal dieser (noch) stark plausibilitätsbegründeten und kaum empirisch untersuchten Unterrichtsformen teilt im deutschsprachigen Raum auch die projektartige Lernund Arbeitsform. Meist wird der Ursprung der Projektmethode vorschnell auf Deweys Konzept der Erfahrung zurückgeführt, ohne die Tiefenstruktur und Vieldimensionalität 
von Deweys Erfahrungsbegriff auszuloten (Combe \& Gebhard 2007). Wir gehen davon aus, dass das Projektlernen in seiner Verlaufslogik erst von einer Irritation und Phantasie würdigenden Erfahrungstheorie aufgeschlossen werden kann; denn das Projektlernen nimmt die Idee eines irritationsbedingten Prozesses auf, institutionalisiert gleichsam den Zyklus von Krise und Gelingen und die phantasievolle Umschichtung der Gegebenheiten und Konstellationen von Problem und Problemlösung in einer Verlaufsfigur (Combe, Leue-Schack \& Pingel 2000; Arnold et al. 2000; Bastian 2007).

Solche Erfahrungsspiralen zwischen Krise, Widerstand, Phantasie und der Bemühung um Ausdruck und Realisierung tauchen im Projektverlauf mehrfach auf. Immer wieder treten Schwierigkeiten auf, die gefunden, formuliert und probeweise gelöst werden müssen, um dabei möglicherweise wieder neue Fragen und Herausforderungen aufzuwerfen. Die für die Phantasie so typische Figur des ,rückgreifenden Vorgriffs“ (s.o.) spielt also auch bei der Projektmethode eine zentrale Rolle. So kommt es zu einem für das funktionale Verständnis der Phantasie als „Text“ (s.o.) charakteristischen Ineinandergreifen von Konstruktion und Rekonstruktion. Es kommt zum fortwährenden Generieren von zukunftsoffenen Hypothesen, die je neu zu den einlaufenden Rückmeldungen ins Verhältnis gesetzt werden müssen.

Bestand die Anfangssituation des Projekts im Entwerfen, so beruht die analog strukturierte Bewegung in der Abschlussphase eher auf einer Rekonstruktion. Vom Überwinden der anfänglichen Krisen geht eine tiefe Bestätigung aus. Die Erfahrungsgeschichte der Lernenden hat sich angereichert, sodass die Dramaturgie des Erfahrungsprozesses ,ihre Vollendung nicht in einem abschließenden Wissen, sondern in jener Neuheit der Erfahrung (findet), die durch die Erfahrung selbst freigespielt wird“ (Gadamer 1960, S. 338 ff.). Freigespielt wird im optimalen Fall also die Offenheit für neue Erfahrungen. Die Erfahrung des Einzelnen, dass er solche Erfahrungskrisen zu bewältigen vermag und um ihre Transformationsvorgänge weiß, kann sich zu einer neuen, offenen, ideensuchenden Haltung zur Welt, zu Anderen und zu sich selbst entwickeln. Aber auch diese beim Durchlaufen von Projekten bewirkten Einstellungseffekte und Möglichkeiten des Wissens- und Erfahrungsaufbaus müssten empirisch dringend untersucht werden.

Zum Abschluss schildern und prüfen wir nun eine Zugangsweise zum Lerngegenstand, die die Möglichkeiten von Erfahrungen in schulischen Lernprozessen - so der Untertitel dieses Aufsatzes - in besonderer Weise verkörpert. Auch hier kommt es zu einer irritationsbedingten Auslösung von Erfahrungsprozessen.

\section{Der Ansatz der Alltagsphantasien als Prototyp des Erfahrungslernens: Unterricht und der andere Umgang mit Irritation und Phantasie}

Die Leistung von Lerntheorien besteht nicht nur darin, Aussagen über Gesetzmäßigkeiten, Typen und Muster menschlichen Lernens zu machen, sondern auch darin, Aussagen über die Beziehung des Menschen zur Welt und zu sich selbst zu machen. Aus unserer Sicht einer Erfahrungstheorie des Lernens gilt es, und das ist eine Summe dieser Ausführungen, den Bezug zu Lerngegenständen so zu ermöglichen, dass sich an ihnen innere Seelenzustände abbilden können. „Der Stoff, an dem ich meine Seele übe“, sagte Alexander von Humboldt dazu. Auf diese Weise wird eine Art von Erfahrung ermöglicht, die 
ihren Namen verdient, weil Erfahrungen in den Blick geraten, die eine innere Bewegung in Beziehung zu einem Lerngegenstand ermöglichen. So werden persönlichkeitswirksame Lernprozesse denkbar und möglich. Der Übergang bzw. die Vermittlung zwischen der Logik der Wissenschaft und der Logik der Erfahrung sowie der Phantasie erfordert eine didaktische Haltung, die die Phantasien und Konnotationen, die Symbolisierungen, die ein Lerngegenstand auslöst, nicht als unpassendes Ornament des eigentlichen Lernstoffes denunziert (Gebhard 2003). Schon unter diesem Blickwinkel darf das Konzept der Alltagsphantasien, das wir zum Abschluss darstellen wollen, als Prototyp des Erfahrungslernens gelten.

Das didaktische Konzept „Alltagsphantasien“ (Gebhard 2007) zielt auf ein vertiefendes Verständnis der individuellen Aneignungs- und Bewertungsprozesse in der Auseinandersetzung mit fachlichen Inhalten und stützt sich neben den soeben ausgeführten theoretischen Zusammenhängen auf subjektorientierte Ansätze der Vorstellungs- und Interessensforschung, die die Bedeutsamkeit individueller Zugänge und Verarbeitungsprozesse hervorheben. Wir werden uns im vorliegenden Zusammenhang fragen müssen, ob und inwiefern hier ein irritierendes Moment vorliegt und produktiv genutzt werden kann. Das Konzept der Alltagsphantasien ist im Bereich der Naturwissenschaftsdidaktik entwickelt und auch empirisch untersucht worden. Themen beispielsweise der modernen Molekularbiologie, die an den „Kern“ des Lebens und der lebendigen Natur rühren, können ein reichhaltiges Spektrum an Vorstellungen, Hoffnungen und Ängsten aktivieren. Dieses Spektrum aktivierter Kognitionen umfasst sowohl explizite Vorstellungen, die im Fokus der Aufmerksamkeit liegen und die sprachlich artikuliert werden können, als auch implizite Vorstellungen, die sich in Form von Assoziationen, Intuitionen oder emotionalen Reaktionen äußern. Diese „Phantasien“, die eine andere Qualität haben als die in der Fachdidaktik anvisierten Alltagsvorstellungen, sind mit dem Begriff der „Alltagsphantasien“ gemeint. Das Konzept der „Alltagsphantasien“ bezieht sich damit auch auf psychoanalytische Positionen und auch auf die aktuelle Diskussion zum Einfluss unbewusster, intuitiver und emotionaler Reaktionen auf alltägliches Denken und Handeln. Analog zur Unterscheidung in bewusste und unbewusste Prozesse in der Psychoanalyse unterscheidet auch die moderne Sozialpsychologie zwei Verarbeitungsmodi des kognitiven Systems: kontrollierte (reflektierende) und assoziative (intuitive) Verarbeitungsprozesse (Goschke \& Bolte 2002; Haidt 2001; Strack \& Deutsch 2004). Alltagsphantasien verweisen damit auch auf eine bislang nur selten thematisierte Ausgangssituation des Lernens: Lernen hat danach - schon lange bevor wir sprachlich und explizit reagieren - in einem unbewusst informationsverarbeitenden System immer schon begonnen.

Alltagsphantasien gehen zum Teil weit über die jeweils thematisierte fachliche Dimension hinaus, sie treten eher als implizites denn als explizites Wissen in Erscheinung und nehmen aufgrund ihrer Bedeutungstiefe (sie beinhalten Aspekte des Selbst-, Menschenund Weltbildes) Einfluss auf Werthaltungen, Interessen und Verhaltensweisen (Gebhard 2004, 2007; Gebhard \& Mielke 2003; Born \& Gebhard 2005). Sie zeigen sich z. B., wenn von „Monstertomaten“ die Rede ist, die an den Mythos des entfesselten Wissenschaftlers Frankenstein erinnern, oder wenn Jugendliche gentechnische Verfahren verurteilen, weil sie diese als unnatürlich empfinden. Gerade die letztgenannte Alltagsphantasie (,Natur als sinnstiftende Idee"), die beim Nachdenken über gentechnische Fragen immer wieder 
auftaucht, zeigt die Verbindung zu Menschen- und Weltbildaspekten. Zwei Beispiele aus Schülerdiskussionen mögen dies verdeutlichen:

- „Man soll der Natur nicht ins Handwerk pfuschen.“

- „Ich habe gerade das Bild von Tieren im Kopf, ich weiß nicht, also wenn jetzt eine Tigermama ein Tigerbaby kriegt. Also sie kriegt vier Stück und eins davon ist blind oder so, dann stößt sie es doch auch weg. Und ich weiß nicht, ich mein, das ist Natur und dem Menschen ist es halt selber überlassen und ich schätz mal nicht, dass es unbedingt negativ ist."

In unseren Untersuchungen, bei denen Alltagsphantasien in Gruppendiskussionen von Jugendlichen empirisch rekonstruiert wurden (Gebhard 2004, 2007; Gebhard \& Mielke 2003), hat sich gezeigt, dass Alltagsphantasien in hohem Maße mit Menschen- und Weltbildern korrelieren. Solche Menschen- und Weltbilder sind bekanntlich ontogenetisch im Kontext eines sehr spezifischen Sozialisationmilieus erworben (Oerter 1999). Alltagsphantasien aktivieren seitens der Schüler ein implizites, kulturelles Wissen und eine ihrem gesellschaftlich-kulturellen Umfeld entsprechende Wahrnehmung. Durch die explizite Thematisierung der Alltagsphantasien, die ein Lerngegenstand hervorruft, kann ein Bezug zwischen fachlichem Wissen, lebensweltlichen Vorstellungen und kulturellen Bildern begünstigt werden. Diese kulturellen Bilder können in Fortsetzung des unterrichtlichen Dialogs immer wieder aktiviert und im Verhältnis zur wissenschaftlichen, fachsprachlichen Deutung qualifiziert werden.

Aber Alltagsphantasien verweisen auch auf den komplexen und irritierenden Beginn einer Beschäftigung mit einem (neuen) Thema. Damit sind wir bei der Frage der Verbindung zu den Prozesskategorien von Erfahrung angelangt. Wir möchten umreißen, inwiefern die schulische Arbeit mit Alltagsphantasien in den Prozessformen des Erfahrungen-Machens erfolgt. Wir können dabei auf Interventionsstudien, eine experimentelle Laboruntersuchung im Rahmen eines DFG-Projekts und, was die Prozessmuster anlangt, auch auf eine fallrekonstruierende Studie zurückgreifen.

Es zeigt sich: Ein solcher Anfang, bei dem Alltagsphantasien erhoben, explizit gemacht und mit den Schülern in ihren differenten Perspektiven kommuniziert werden, irritiert. Das haben wir sowohl im Kontext einer laborexperimentellen Studie (Oschatz, Gebhard \& Mielke 2010) gefunden als auch in einer inhaltsanalytisch ausgewerteten Fallstudie (Combe \& Gebhard 2009). Die primäre Wirkung der Alltagsphantasien kann insofern als eine Irritation beschrieben werden, die zunächst von der routinierten und effizienten Beschäftigung mit einer Thematik wegführt. Allerdings - und das konnten wir in Interventionsstudien empirisch zeigen - lohnt sich diese irritierende Tiefe: Wenn die Phantasien willkommen sind, wenn sie immer wieder zum Gegenstand expliziter Reflexion gemacht werden - auch wenn sie abschweifig sind - wird ein Unterricht, der Alltagsphantasien berücksichtigt, sinnhafter erlebt, unterstützt die Motivation und ist auch im Hinblick auf den kognitiven Lernprozess - langfristig, meist schon mittelfristig - effizienter (Born 2007; Born \& Gebhard 2005; Monetha 2009; Monetha \& Gebhard 2008).

In der Fallstudie, die die Nutzung der Alltagsphantasien im Unterrichtsprozess untersucht, zeigt sich, dass ein solcher Beginn als ambivalent empfunden wird. Einerseits betrachten Schüler diesen Zugang als nicht „legitim“, ja als befremdlich. Andererseits aber nehmen sie positiv wahr, dass sie als partizipationsfähige Teilnehmer des Unterrichts 
angesprochen werden. Das Thema des Unterrichts wird mit einem persönlichen Thema versehen und die Schüler situieren sich innerhalb eines Gegenstandsbereichs. Man schafft gleichsam einen Vorposten in einem ansonsten noch relativ fremden Gelände. Zudem ist dieser Anfang von besonderer suggestiver Präsenz, denn es handelt sich bei diesem Setting um ein Aufbrechen eingefahrener Handlungskreisläufe, kurz: um eine typische Krise der Routine. Dadurch werden die Sperren und Widerstände umgangen, die auftreten können, wenn die fachlichen Anforderungen ,pur“ und unvermittelt an die Schüler herangetragen werden.

Doch die Irritation hat auch inhaltliche Gründe. Immer wieder neue Irritationspunkte ergeben sich dadurch, dass die Spannung zwischen Alltagsphantasien und fachsprachlicher Deutung (siehe Abschnitt 3.2) gerade nicht ausgeklammert wird. In der weiteren Verlaufslogik des Unterrichts ergibt sich eine Pendelbewegung, die sich zwischen der sachlichen und der subjektiven Seite bewegt; ein Wechselspiel, in dem immer wieder auf die vergegenwärtigten Muster der Alltagsphantasien zurückgekommen werden kann, sodass durch die Konfrontation von alläglichen Phantasiemustern mit fachlichen Deutungsmustern neue irritationssensible Zonen entstehen. Somit ist ersichtlich, dass die Schüler durch die Thematisierung von Alltagsphantasien sowohl kognitiv als auch affektiv in besonderer Weise herausgefordert werden. Ein solcher Unterricht wird als Erfahrungszusammenhang abgegrenzt: Als eine mit der Irritation des bisher Selbstverständlichen verbundene Handlungs-, Erlebnis- und Reflexionseinheit.

Dabei ist es wichtig, wie mit den Alltagsphantasien umgegangen, wie an sie angeknüpft wird. Dieses Anknüpfen, Weiterverarbeiten, Strukturieren und Zurückkommen verlangt bisweilen einen (textlichen) Niederschlag. Die Schüler beginnen, ihre Phantasien zu sortieren und auszudiskutieren. Oft wird man diese zeitlich und sachlich aufwändige Annäherung an einen Gegenstand als verlorene Zeit ansehen. Aber die Phantasie bricht die Fremdheit des Gegenstands auf, zerlegt ihn und fügt ihn nach eigenen Gesetzen wieder zusammen. In Bezug auf die Nutzung der Alltagsphantasien zeigt sich, dass diese ihre eigene Zähigkeit haben. Zugleich aber bilden sie eine Plattform, von der aus neue Legierungen zwischen dem Alten und dem Neuen, zwischen der fachlichen und der alltäglichen Sicht möglich werden. So kann gesagt werden, dass Alltagsphantasien ,wie ein Gerüst von Stützbalken das im Diskurs konstruierte Objekt tragen“ (Wagner 1994, S. 159). Um die Rekonstruktion und Konstruktion dieser „Stützbalken“ geht es beim Ansatz der Alltagsphantasien. Diese Stützbalken sind für Bildungsprozesse deshalb besonders wichtig, weil sie die kulturellen und sozialen Konzepte, unsere impliziten Welt- und Menschenbilder transportieren. Zudem machen sie als Intuitionen natürlich nicht vor Fächergrenzen halt und deren explizite Reflexion sichert den fachübergreifenden Ansatz (Decke-Cornill \& Gebhard 2007).

Das Beispiel der Alltagsphantasien zeigt, wie in einem irritationsbedingten Prozess eine Erfahrungsbewegung zwischen Ich und Gegenstand in Gang gesetzt werden kann. Es reicht also nicht, wenn Lernenden die Dinge nur in ihrer objektivierenden Variante beigebracht werden. Eine geradlinige objektivierende Sicht der Dinge unterschlägt die subjektivierenden Schattierungen, entsinnlicht die Phänomene, grenzt den subjektiv gemeinten Sinn aus und bringt die Dinge den Subjekten nicht nahe. Objekte der Außenwelt haben nicht nur eine Bedeutung als objektive Gegebenheiten, sondern auch eine symbolische Bedeutung, in der persönliche Erfahrungen, Beziehungen und Phantasien, Narrationen 
zusammenfließen (Gebhard 1992). In der Vermittlung zwischen beiden Zugängen besteht die Chance, einer an sich unbegreiflichen Welt (Blumenberg 1981) Sinn zu verleihen bzw. diese als sinnhaft zu erleben. Wir haben gezeigt, wie in einem irritationsbedingten Prozess über die vermittelnde Repräsentationsqualität der Phantasie eine Austauschbewegung zwischen Ich und Gegenstand in Gang gesetzt werden kann, die den Namen Erfahrung verdient. Eine didaktische Haltung, die dazu passt, lädt ein zum Phantasieren, hat Zeit und Muße zum Verweilen und gibt damit dem Aufbau von Erfahrung und Sinn eine Chance.

Open Access Dieser Artikel unterliegt den Bedingungen der Creative Commons Attribution Noncommercial License. Dadurch sind die nichtkommerzielle Nutzung, Verteilung und Reproduktion erlaubt, sofern der/die Originalautor/en und die Quelle angegeben sind.

\section{Literatur}

Arnold, E., Bastian, J., Combe, A., Schelle, C., \& Reh, S. (2000). Schulentwicklung und Wandel der pädagogischen Arbeit. Hamburg: Bergmann und Helbig.

Bannert, M., \& Schnotz, W. (2006). Vorstellungsbilder und Imagery-Strategien. In H. Mandl \& H. F. Friedrich (Hrsg.), Handbuch Lernstrategien (S. 72-88). Göttingen: Hogrefe.

Bastian, J. (2007). Einführung in die Unterrichtsentwicklung. Weinheim: Beltz.

Bastian, J., Combe, A., \& Langer, R. (2003). Feedback-Methoden. Weinheim: Beltz.

Baumert, J. (2008). Was soll man unter Bildung verstehen? Die Deutsche Schule 100 (1), 16-21.

Baumert, J., Lehmann, R., Lehrke, M., Schmitz, B., Clausen, M., Hosenfeld, I., et al. (1997). TIMSS - mathematisch-naturwissenschafticher Unterricht im internationalen Vergleich. Deskriptive Befunde. Opladen: Leske + Budrich.

Benjamin, W. (1977). Der Erzähler. Betrachtungen zum Werk Nikolai Lesskows. In W. Benjamin (Hrsg.), Gesammelte Schriften (Bd. II., S. 438-465). Frankfurt a.M.: Suhrkamp.

Benner, D. (2005). Einleitung. Über pädagogisch relevante und erziehungswissenschaftlich fruchtbare Aspekte der Negativität menschlicher Erfahrung. In D. Benner (Hrsg.), Erziehung - Bildung - Negativität (Zeitschrift für Pädagogik: Beiheft 49, S. 7-23). Weinheim: Beltz.

Bittner, G. (1998). Metaphern des Unbewußten. Eine kritische Einführung in die Psychoanalyse. Stuttgart: Kohlhammer.

Blumenberg, H. (1981). Die Lesbarkeit der Welt. Frankfurt a.M.: Suhrkamp.

Blumenberg, H. (1987). Das Lachen der Thrakerin. Eine Urgeschichte der Theorie. Frankfurt a.M.: Suhrkamp.

Bollnow, O. F. (1968). Der Erfahrungsbegriff in der Pädagogik. Zeitschrift für Pädagogik, 14, 221-252.

Born, B. (2007). Zum Einfluss von Alltagsphantasien auf das Lernen. Studien zur Schul- und Bildungsforschung. Wiesbaden: VS Verlag für Sozialwissenschaften.

Born, B., \& Gebhard, U. (2005). Intuitive Vorstellungen und explizite Reflexion: Zur Bedeutung von Alltagsphantasien bei Lernprozessen zur Bioethik. In B. Schenk (Hrsg.), Bausteine einer Bildungsgangtheorie (S. 255-271). Wiesbaden: VS Verlag für Sozialwissenschaften.

Buck, G. (1967). Lernen und Erfahrung, Epagogik. Darmstadt: Wissenschaftliche Buchgesellschaft.

Casey, C. (1996). Incorportating cognitive apprenticeship in multi-media. Educational Technology Research and Development, 44, 71-84.

Cognition and Technology Group at Vanderbilt (1997). The Jasper project. Lessons in curriculum, instruction, assessment and professional development. New York: Lawrence Erlbaum. 
Collins, A., Brown, J. S., \& Newman, S. E. (1989). Cognitive apprenticeship. Teaching the crafts of reading, writing and mathematics. In L. B. Resnick (ed.), Knowing, learning and instruction. Essays in honor of Robert Glaser (S. 453-494). New York: Lawrence Erlbaum.

Combe, A. (1992). Bilder des Fremden. Romantische Kunst und Erziehungskultur. Opladen: Westdeutscher Verlag.

Combe, A. (2004). Brauchen wir eine Bildungsgangforschung? In T. Trautmann (Hrsg.), Entwicklungsaufgaben im Bildungsgang (Bildungsgangforschung, Bd. 6, S. 48-68). Wiesbaden: VS Verlag für Sozialwissenschaften.

Combe, A. (2005). Lernende Lehrer - Professionalisierung und Schulentwicklung im Lichte der Bildungsgangforschung. In B. Schenk (Hrsg.), Bausteine einer Bildungsgangtheorie (S. 6990). Wiesbaden: VS Verlag für Sozialwissenschaften.

Combe, A. (2006). Hatten die schon Schuhe? Zur Theorie des Erfahrungslernens. Pädagogik, 58 (6), 32-36.

Combe, A., \& Gebhard, U. (2007). Sinn und Erfahrung. Zum Verständnis fachlicher Lernprozesse in der Schule. Opladen: Barbara Budrich.

Combe, A., \& Gebhard, U. (2009). Alltagsphantasien als erfahrungsbezogene Annäherung an den Lerngegenstand. Hamburg: Bisher unveröffentlichtes Manuskript.

Combe, A., Leue-Schack, K., \& Pingel, K. (2000). Unterrichtsentwicklung. In E. Arnold et al. (Hrsg.), Schulentwicklung und Wandel der pädagogischen Arbeit (S. 35-78). Hamburg: Bergmann und Helbig.

Copei, F. (1969). Der fruchtbare Moment im Bildungsprozess. Heidelberg: Quelle Meyer.

Decke-Cornill, H. \& Gebhard, U. (2007). Ästhetik und Wissenschaft. Zum Verhältnis von literarischer und naturwissenschaftlicher Bildung. In L. Brellade \& W. Haller (Hrsg.), Literaturunterricht, Kompetenzen und Bildung(S. 11-30). Trier: WVT.

Dewey, J. (1988). Kunst als Erfahrung. Frankfurt a.M.: Suhrkamp.

Dewey, J. (2002). Wie wir denken. Mit einem Nachwort neu hrsg. von R. Horlacher \& J. Oelkers. Zürich: Pestalozzianum.

Dewey, J. (2007). Erfahrung und Natur. Frankfurt a.M.: Suhrkamp.

Duit, R. (1993). Schülervorstellungen - von Lerndefiziten zu neuen Unterrichtsansätzen. Naturwissenschaften im Unterricht - Physik, 4 (16), 4-10.

Duit, R. (1996). Lernen als Konzeptwechsel im naturwissenschaftlichen Unterricht. In R. Duit \& C. v. Rhöneck (Hrsg.), Lernen in den Naturwissenschaften (S. 145-162). Kiel: IPN.

Duit, R. (2006). Schülervorstellungen und das Lernen von Physik. In R. Girwitz, M. Gläser-Zikuda, M. Laukenmann \& T. Rubitzko (Hrsg.), Lernen im Physikunterricht (Festschrift für Christoph von Rhöneck, S. 13-22). Hamburg: Dr. Kovać.

Eikenbusch, G. (Hrsg.) (2008): „Aufgabenkultur“ (Thementeil). Pädagogik, 60 (3).

English, A. (2005). Negativität der Erfahrung. Pragmatismus und die Grundstruktur des Lernens. Erziehungswissenschaftliche Reflexionen zur Bedeutung des Pragmatismus von Peirce, James und Mead für Deweys Theorie der reflective experience. In D. Benner (Hrsg.), Erziehung - Bildung - Negativität (Zeitschrift für Pädagogik: Beiheft 49, S. 49-61). Weinheim: Beltz.

Foucault, M. (1996). Der Mensch ist ein Erfahrungstier. Gespräch mit Ducio Trombardori. Frankfurt a.M.: Suhrkamp.

Gadamer, H. G. (1960). Wahrheit und Methode. Grundzüge einer philosophischen Hermeneutik. Gesammelte Werke (Bd. 1). Tübingen: Mohr Siebeck.

Gebhard, U. (1992). Träumen im Biologieunterricht? - Psychoanalytische Betrachtungen zu unbewußten Einflüssen auf das Denken. Unterricht Biologie, 172, 44-46.

Gebhard, U. (1999). Weltbezug und Symbolisierung: Zwischen Objektivierung und Subjektivierung. In Gesellschaft für Didaktik des Sachunterrichts (Hrsg.), Probleme und Perspektiven des Sachunterrichts. Bd. 9: Umwelt, Mitwelt, Lebenswelt im Sachunterricht (S. 33-53). Bad Heilbrunn: Klinkhardt. 
Gebhard, U. (2003). Die Sinndimension im schulischen Lernen: Die Lesbarkeit der Welt. Grundsätzliche Überlegungen zu Lernen und Lehren im Anschluss an PISA. In B. Moschner, H. Kiper \& U. Kattmann (Hrsg.), PISA 2000 als Herausforderung. Baltmannsweiler: Schneider Hohengehren.

Gebhard, U. (2004). Menschenbilder von Jugendlichen angesichts der gentechnischen Herausforderung. In U. Gerber \& H. Meisinger (Hrsg.), Das Gen als Maß aller Menschen. Menschenbilder im Zeitalter der Gene (S. 79-100). Frankfurt a.M.: Peter Lang.

Gebhard, U. (2005). Symbole geben zu denken. Sprache und Verstehen im naturwissenschaftlichen Unterricht. Plädoyer für das Philosophieren im naturwissenschaftlichen Unterricht. In C. Hößle \& K. Michalik (Hrsg), Philosophieren mit Kindern und Jugendlichen. Didaktische und methodische Grundlagen des Philosophierens (S. 48-59). Baltmannsweiler: Schneider Hohengehren.

Gebhard, U. (2007). Intuitive Vorstellungen bei Denk und Lernprozessen: Der Ansatz der „Alltagsphantasien“. In D. Krüger \& H. Vogt (Hrsg.), Theorien in der biologiedidaktischen Forschung (S. 117-128). Berlin: Springer.

Gebhard, U. \& Mielke, R. (2003). „Die Gentechnik ist das Ende des Individualismus“: Latente und kontrollierte Denkprozesse bei Jugendlichen. In D. Birnbacher \& E. Martens (Hrsg), Philosophie und ihre Vermittlung (S. 202-218). Hannover: Siebert.

Goschke, T. \& Bolte, A. (2002). Emotion, Kognition und Intuition: Implikationen der empirischen Forschung für das Verständnis moralischer Urteilsprozesse. In S. A. Döring \& V. Mayer (Hrsg.), Die Moralität der Gefühle (Deutsche Zeitschrift für Philosophie, Sonderbd. 4, S. 39-57). Berlin: Akademie-Verlag.

Gudjons, H. (2003). Didaktik zum Anfassen. Bad Heilbrunn: Klinkhardt.

Haidt, J. (2001). The emotional dog and its rational tail: A social intuitionist approach to moral judgement. Psychological Review, 108, 814-834.

Hascher, T. (2005). Emotionen im Schulalltag: Wirkungen und Regulationsformen. Zeitschrift für Pädagogik, 51, 610-625.

Helmke, A. (2003). Unterrichtsqualität erfassen, bewerten, verbessern. Seelze: Kallmeyer.

Hiebert, J., Gallimore, R., Garnier, K., Givvin, K. B., Hollingsworth, H., Jacobs, J., et al. (2003). Teaching mathematics in seven countries. Results from the TIMMS 1999 video study. Washington: National Center for Education Statistics.

Hoffmann, S. O. (1987). Die psychoanalytische Abwehr - aktuell, antiquiert oder obsolet. Forum der Psychoanalyse, 3, 22-39.

Kambartel, F. (1972). Erfahrung. In J. Ritter (Hrsg.), Historisches Wörterbuch der Philosophie, (Bd. 2, S. 609-617). Basel: Schwabe.

Kattmann U. \& Gropengießer, H. (1996). Modellierung der didaktischen Reduktion. In R. v. Duit \& C. V. Rhönede. (Hrsg.), Lernen in den Naturwissenschaften (S. 180-204). Kiel: IPN.

Klieme, E. (2006). Empirische Unterrichtsforschung: Aktuelle Entwicklungen, theoretische Grundlagen und fachspezifische Befunde. Zeitschrift für Pädagogik, 52, 765-773.

Lüders, M. (2003). Unterricht als Sprachspiel. Eine systematische und empirische Studie zum Unterrichtsbegriff und zur Unterrichtssprache. Bad Heilbrunn: Klinkhardt.

Meyer-Drawe, K. (1996). Vom anderen lernen. Phänomenologische Betrachtungen in der Pädagogik. In M. Borelli \& J. Ruhloff (Hrsg.), Deutsche Gegenwartspädagogik (Bd. 2, S. 85-98). Baltmannsweiler: Schneider Hohengehren.

Meyer-Drawe, K. (2005). Lernen als Erfahrung. Zeitschrift für Erziehungswissenschaft, 6, 505-514.

Mollenhauer, K. (1996). Grundfragen ästhetischer Bildung. Weinheim: Juventa.

Monetha, S. (2009). Alltagsphantasien, Motivation und Lernleistung. Opladen: Budrich.

Monetha, S., \& Gebhard, U. (2008). Alltagsphantasien, Sinn und Motivation. In H.-C. Koller (Hrsg.), Sinnkonstruktion und Bildungsgang. Zur Bedeutung individueller Sinnzuschreibungen im Kontext schulischer Lehr-Lern-Prozesse (S. 65-86). Opladen: Budrich. 
Neber, H. (2004). Förderung epistemischen Fragens im Religionsunterricht. Unterrichtswissenschaft, 32, 308-320.

Neber, H. (2006). Fragenstellen. In H. Mandl \& H. F. Friedrich (Hrsg.), Handbuch Lernstrategien (S. 50-58). Göttingen: Hogrefe.

Niegemann, H. M. (2004). Lernen und Fragen: Bilanz und Perspektiven der Forschung. Unterrichtswissenschaft, 32, 345-356.

Nietzsche, F. (1966). Werke I bis III. Hrsg. von K. Schlechta. München: Hanser.

Oerter, R. (Hrsg.) (1999). Menschenbilder in der modernen Gesellschaft. Konzeptionen des Menschen in Wissenschaft, Bildung, Kunst, Wirtschaft und Politik. Stuttgart: Lucius \& Lucius.

Oettingen G. (1997). Psychologie des Zukunftsdenkens: Erwartungen und Phantasien. Göttingen: Hogrefe.

Oettingen, G., Pak, H., \& Schnetter, K. (2001). Self-Regulation of goal setting: Turning free fantasies about future into binding goals. Journal of Personality and Social Psychology, 80, $736-353$.

Oevermann, U. (1991). Genetischer Strukturalismus und das sozialwissenschaftliche Problem der Entstehung des Neuen. In S. Müller-Dohm (Hrsg.), Jenseits der Utopie (S. 267-338). Frankfurt a.M.: Suhrkamp.

Oevermann, U. (1996). Theoretische Skizze einer revidierten Theorie professionalisierten Handelns. In: A. Combe \& W. Helsper (Hrsg.), Pädagogische Professionalität. Untersuchungen zum Typus pädagogischen Handelns (S. 70-182). Frankfurt a.M.: Suhrkamp.

Oevermann, U. (2004). Sozialisation als Prozess der Krisenbewältigung. In D. Geulen \& H. Veit (Hrsg.), Sozialisationstheorie interdisziplinär (S. 155-181). Stuttgart: Lucius \& Lucius.

Oschatz, K., Gebhard, U., \& Mielke, R. (2010). Irritation als Chance. Auswirkungen intuitiver Vorstellungen auf das Lernen über Gentechnik. In D. Krüger (Hrsg.), Erkenntnisweg Biologiedidaktik. Kassel: Universität.

Prinz, W. (1983). Wahrnehmung und Tätigkeitssteuerung. Berlin: Springer.

Rehm, M. (2003). Über die Chancen und Grenzen ,,moralischer “ Erziehung im naturwissenschaftlichen Unterricht. Berlin: Logos.

Reinfried, S. (2007). Alltagsvorstellungen und Lernen im Fach Geographie am Beispiel des Conceptual Change. Geographie und Schule, 29 (165), 19-28.

Ritter, J. (Hrsg.) (1971). Historisches Wörterbuch der Philosophie. Basel: Schwabe.

Rumpf, H. (2004). Diesseits der Belehrungswut. Weinheim: Juventa.

Sartre, J. P. (1971). Das Imaginäre. Phänomenologische Psychologie der Einbildungskraft. Reinbek: Rowohlt.

Schäfer, G. (1986). Spiel, Spielraum, Verständigung. Untersuchungen zur Entwicklung von Spiel und Phantasie im Kindes- und Jugendalter. Weinheim: Juventa.

Schäfer, G. (1995). Bildungsprozesse im Kindesalter. Selbstbildung, Erfahrung und Lernen in der frühen Kindheit. Weinheim: Juventa.

Seel, N. M. (2000). Psychologie des Lernens. München: Reinhardt.

Strack, F., \& Deutsch, R. (2004). Reflective and Impulsive Determinants of Social Behavior. Personality and Social Psychology Review, 8, 220-247.

Stiegler, J. W., \& Hiebert, J. (1999). The teaching gap. New York: Free Press.

Wagner, W. (1994). Alltagsdiskurs. Die Theorie Sozialer Repräsentation. Göttingen: Hogrefe.

Waldenfels, B. (2002). Bruchlinien der Erfahrung. Frankfurt a.M.: Suhrkamp.

Waldenfels, B. (2004). Phänomenologie der Aufmerksamkeit. Frankfurt a.M.: Suhrkamp.

Winnicott, D. W. (1995): Übergangsobjekte und Übergangsphänomene. In D. W. Winnicott (Hrsg.), Vom Spiel zur Kreativität (S. 10-36). Stuttgart: Klett-Cotta.

Ziehe, T. (1996). Vom Preis selbstbezüglichen Wissens. In A. Combe \& W. Helsper (Hrsg.), Pädagogische Professionalität. Untersuchungen zum Typus pädagogischen Handelns (S. 924-942). Frankfurt a.M.: Suhrkamp. 